\title{
Balance Deficit and Brain Connectivity in Children with Attention-Deficit/Hyperactivity Disorder
}

\author{
Sun Mi Kim¹, Gi Jung Hyun'1, Tae-Woon Jung², Young Don Son ${ }^{3}$, In-Hee Cho ${ }^{4}$, \\ Baik Seok Kee ${ }^{1}$, and Doug Hyun $\mathrm{Han}^{1}{ }^{凶}$ \\ ${ }^{1}$ Department of Psychiatry, Chung-Ang University Hospital, Seoul, Republic of Korea \\ ${ }^{2}$ Department of Therapeutic Riding, Jeonju Kijeon College, Jeonju, Republic of Korea \\ ${ }^{3}$ Department of Biomedical Engineering, Gachon University Gil Medical Center, Incheon, Republic of Korea \\ ${ }^{4}$ Dr. Cho's Child \& Adolescent Psychiatric Clinic, Seongnam, Republic of Korea
}

Objective We aimed to assess disturbances in postural and gait balance and functional connectivity within the brain regions controlling balance in children with attention-deficit/hyperactivity disorder (ADHD).

Methods Thirteen children with ADHD and 13 age- and sex-matched controls were recruited. Gait balance was assessed by the difference in the center of pressure (COP) between the left and right foot, as well as the difference in plantar pressure between the left and right foot during gait. Neuroimaging data were acquired using a 3.0 Tesla MRI scanner. Functional connectivity between the vermis of the cerebellum and all other brain regionswas assessed.

Results The difference in plantar pressure between the left foot and right foot in the ADHD group was greater than that observed in the control group. The average COP jerk score of the right foot in the ADHD group was higher than that observed in the control group. A higher functional connectivity between the cerebellum and the right middle frontal gyrus (premotor cortex) and medial frontal gyrus (cingulate gyrus) was observed in the control group relative to the ADHD group. In the ADHD group, the difference in plantar pressure between the left and right foot was also negatively correlated with the beta-value within the middle frontal gyrus.

Conclusion Children with ADHD had disturbance of balance as assessed by plantar pressure. Decreased brain connectivity from the cerebellum to the premotor cortex and anterior cingulate was associated with disturbances of posture and balance in children with ADHD.

Psychiatry Investig 2017;14(4):452-457

Key Words Attention-deficit/hyperactivity disorder, Gait balance, Resting-state functional magnetic resonance imaging, Cerebellum, Middle frontal gyrus.

\section{INTRODUCTION}

\section{Attention-deficit/hyperactivity disorder and gait disturbances}

Attention-deficit/hyperactivity disorder (ADHD) is one of the most common developmental disorders in children and adolescents. ${ }^{1,2}$ The primary cognitive symptom of ADHD consists of in attention, while the primary behavioral symptoms include hyperactivity and impulsive behavior. ${ }^{3}$ There has been

Received: February 4, 2016 Revised: May 29, 2016

Accepted: June 30, 2016 Available online: May 16, 2017

$\triangle$ Correspondence: Doug Hyun Han, MD, PhD

Department of Psychiatry, Chung-Ang University School of Medicine, 102 Heukseok-ro, Dongjack-gu, Seoul 06973, Republic of Korea

Tel: +82-2-6299-3132, Fax: +82-2-813-5387, E-mail: hduk@yahoo.com

(c) This is an Open Access article distributed under the terms of the Creative Commons Attribution Non-Commercial License (http://creativecommons.org/licenses/by$\mathrm{nc} / 4.0$ ) which permits unrestricted non-commercial use, distribution, and reproduction in any medium, provided the original work is properly cited. considerable research on cognitive and motor disturbances in children with ADHD., ${ }^{1,2}$ About $50 \%$ of children with ADHD have been reported to show clumsiness in motor performance below the norms for their age. ${ }^{4}$ In a comparison of the Physical and Neurological Examination for Soft Signs Scale (PANESS) scores between 35 medication-naivve boys with ADHD (8-11 years) and 30 age-matched controls, Konicarova et al..$^{5}$ reported that boys with ADHD showed disturbance of postural and gait balance as compared to controls. In contrast, Schlee et al. ${ }^{6}$ reported that the balance and postural capacity of children with ADHD, as measured by plantar foot sensitivity and static balance, showed no significant difference compared to controls.

Brain imaging data from children with ADHD have suggested that motor disturbances such as those in postural and gait balance are associated with impairments in prefrontal functioning (hypofrontality) ${ }^{7}$ and a deficit of cerebellar inhibi- 
tory functions. ${ }^{8}$ Hypofrontality in children with ADHD has been thought to be associated with the delayed maturation of frontal lobe functions of compensatory response within basal ganglia and cerebellum. ${ }^{9,10}$ Other brain imaging studies of ADHD have revealed that volume reductions in the cerebellar vermis are associated with disturbances in postural and gait control. ${ }^{11,12}$

\section{Plantar pressure and gait balance}

Postural stability is thought to be associated with the maintenance of an upright position during quiet standing and with the recovery of balance during voluntary movement. ${ }^{13}$ Postural stabilityis frequently represented by the body's global center of mass (COM). The central nervous system maintains the COM in space and the oscillation of COM is called postural sway. ${ }^{14}$ Postural sway is thought to be related to the shift in the center of pressure (COP) from the anteroposterior (AP) to mediolateral (ML) foot. In addition to the COP, mean plantar pressure of the foot is commonly used for evaluating foot and lower limb function as well as gait balance during walking. ${ }^{15}$ For a reliable assessment of plantar pressure in children 7-11 years of age, Cousins et al. ${ }^{16}$ suggested considering variables including peak pressure, peak force, pressure-time integral and force-time integral.

\section{Hypothesis}

So far, there have been inconsistent study results on gait disturbances in ADHD. In addition, there have been no studies that investigate the direct correlation between postural and gait balance and activity within the brain regions in ADHD, controlling for medication use. Therefore, this study was aimed to assess disturbances in postural and gait balance and functional connectivity with in the brain regions controlling balance in children with ADHD who were medication-naïve or drug-free for 6 months prior to the study. We hypothesized that children with ADHD would exhibit greater balance disturbance in gait and decreased brain connectivity within the fronto-striatal-cerebellar circuit as compared to control children. We also hypothesized that gait disturbance would be associated with hypo-connectivity between the cerebellum and frontal lobe in children with ADHD.

\section{METHODS}

\section{Participants}

Thirteen children with ADHD (9-12 years of age) and 13 age- and sex-matched controls were recruited from the Department of Psychiatry, Kacheon University Hospital. All children were assessed with the Korean Kiddie Schedule for Affective Disorders and Schizophrenia, Present and Lifetime
Version ${ }^{17}$ and diagnosed according to Diagnostic Statistical Manual of Mental Disorder-IV-TR criteria. Inclusion criteria for ADHD included children who: 1) were diagnosed as having ADHD, 2) were medication-naïve or drug-free for 6 months prior to the study, 3) and had an intelligence quotient (IQ) $\geq 80$. Exclusion criteria included children who: 1) had other psychiatric comorbidities including depression, autism spectrum disorder, and tic disorder, 2) had a history of head trauma and substance abuse, or 3) had a contraindication for MRI scanning such as claustrophobia, metal implants, or cerebrovascular accidents. The same contraindications applied to the control children. Finally, 13 ADHD children consisting of $4 \mathrm{ADHD}$ children with predominantly inattentive presentation, $6 \mathrm{ADHD}$ children with predominantly hyperactive/ impulsive presentation, and $3 \mathrm{ADHD}$ children with combined presentation were recruited. In addition, there were no ADHD children who had other psychiatric comorbidities.

The research protocol was approved by the Kacheon University Hospital Institutional Review Board. Written informed consent was provided by the children and their parents.

\section{Measurement of balance using plantar pressure}

Gait balance was assessed by the difference in the COP between the left and right foot and the difference in plantar pressure between the left and right foot during gait. The pressures of the foot were assessed with the TekScanMatScan ${ }^{\circledR}$ system floor mat (Boston, MA, USA), which is $5 \mathrm{~mm}$ thick (432× $368 \mathrm{~mm}$ ) and has 2288 resistive sensors $\left(1.4\right.$ sensors $\left./ \mathrm{cm}^{2}\right)$ and a sampling frequency of $40 \mathrm{Hertz}(\mathrm{Hz})$. To prevent intentional weight bearing on the floor mat, children were asked to squat twice for 10 seconds. During these 10 seconds, the COPs of both feet were estimated and the COP of the last second (50 frames) was recorded for analysis. The smoothness of the COP trajectory in theantero-posterior (AP) to mediolateral (ML) direction within the foot was quantified using normalized jerk scores during gait. ${ }^{18}$ To capture dynamic plantar pressure for a constant gait velocity, a two-step gaitprotocol was used..$^{19}$ After children were familiarized with walking over the floor mat, data was collected at a natural gait pattern with a self-selected speed. The peak pressures $(\mathrm{k} \mathrm{Pa})$ within the seven regions of the foot lateral heel, medial heel, mid-foot, first metatarsophalangeal joint (MPJ), second to fifth MPJ, hallux, and lesser toes ${ }^{16}$ were obtained and the average peak pressure of both feet was calculated. The average peak pressure in addition to the temporal measures of pressuretime integrals $\left(\mathrm{k} \mathrm{Pa.s} / \mathrm{cm}^{2}\right)$ was selected for analysis.

\section{Acquisition and analysis of brain connectivity}

All imaging data were acquired using a 3.0 Tesla Verio MRI scanner (SIEMENS, Erlangen, Germany). Functional image 
parameters were as follows: a gradient-echo planar sequence sensitive to the blood oxygen level-dependent (BOLD) contrast [repetition time $(\mathrm{TR})=3,000 \mathrm{~ms}$, echo time $(\mathrm{TE})=30 \mathrm{~ms}$, and flip angle $=90^{\circ}$, whole brain volumes with $3.5-\mathrm{mm}$-thick transverse slices, a voxel size of $3.5 \times 3.5 \times 3.5 \mathrm{~mm}$, no interslice gap, and in-plane resolution of $64 \times 64$ pixels. Patients were instructed to lie still in the scanner with their eyes closed, but to remain awake. For anatomical imaging, 3D T1-weighted magnetization-prepared rapid gradient echo (MPRAGE) data were collected with the following parameters: $\mathrm{TR}=1,900 \mathrm{~ms}$, $\mathrm{TE}=3.00 \mathrm{~ms}, \mathrm{FOV}=256 \times 256 \mathrm{~mm}, 120$ slices, $1.0 \times 1.0 \times 1.0 \mathrm{~mm}$ voxel size. Images were realigned to Talairach coordinates using the anterior and posterior commissures and the sagittal sulcus plane. Brain Voyager software (BVQX 1.9; Brain Innovation, Maastricht, Netherlands) was used for the analysis of functional images. Functional data was co-registered to the anatomical 3D data sets for each participant using the provided multi-scale algorithms. The 3D structural images were spatially normalized to the standard Talairach space. ${ }^{20} \mathrm{~A}$ nonlinear transformation was applied to the T2-weighted fMRI time series data. Slice scan time and 3D motion correction were applied and the functional data were spatially smoothed using a Gaussian kernel with a full width at half maximum (FWHM) of $6 \mathrm{~mm}$, which was temporally filtered using linear trend removal and Fourier analysis high-pass filtering with a cutoff of 3 cycles of the full time course. Brain connectivity within the fronto-striatal-cerebellar circuit was measured by functional connectivity (FC) analysis using resting state functional magnetic resonance imaging (rs-fMRI) data. RsfMRI allowed us to examine intrinsic brain activity while the participant was at rest. FC is defined asa temporal association between two brain areas that are spatially isolated. FC analysis allowed us to assess the neural circuitry suggestive of connectivity between brain regions that share functional properties. ${ }^{21}$ Functional connectivity between the vermis of the cerebellum and all other regions of the brain was assessed by drawing a cube of $10 \mathrm{~mm}$ on each side, which was centered on the vermis of the cerebellum using Talairach coordinates $(0,-68$, -16) (Figure 1). Children with excessive head movements (greater than $3 \mathrm{~mm}$ in the $\mathrm{x}, \mathrm{y}$ or $\mathrm{z}$ dimensions in length or greater than $3^{\circ}$ around the $\mathrm{x}, \mathrm{y}$ or $\mathrm{z}$ axis in rotation) were tobe excluded from the fMRI analysis. However, no participants were excluded from the analysis based on these criteria.

\section{Statistical analysis}

Demographic characteristics between the ADHD group and control group were analyzed with the Mann-Whitney $U$ test and chi-square test. The average time course of the BOLD signal was extracted from the seed region of the vermis of the cerebellum and used as the model predictor in a general linear model analysis (GLM) to determine temporally related brain regions in the form of individual subject statistical parametric maps (SPM) of temporal correlation coefficients (beta-values). Other predictors were not used in the GLM analysis. A post-hoc region of interest analysis was performed calculating the average beta-values using a mask of the significantly higher FC in the middle frontal and medial frontal gyri in the ADHD group as compared to thecontrol group. For all analyses, we regarded associations as significant when the false discovery rate (FDR) correction was equal to or less than 0.05 .

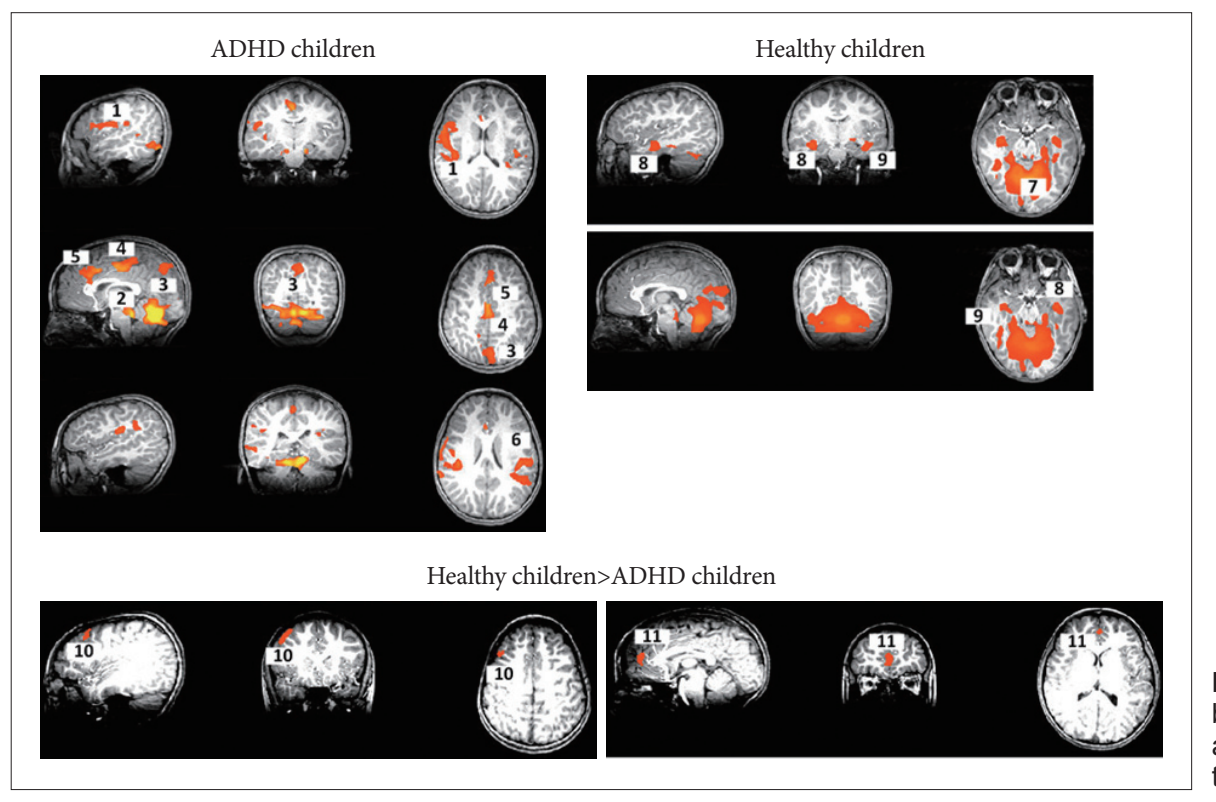

Figure 1. Brain functional connectivity between the vermis of the cerebellum and other brain areas. ADHD: attention-deficit/hyperactivity disorder. 


\section{RESULTS}

\section{Demographic characteristics}

There were no significant differences in age, sex, education years, IQ, body index (height and weight) or children's depressive inventory (CDI) score between the two groups. The mean $\mathrm{K}$-ARS scores in the ADHD group were higher than those in the control group $(\mathrm{z}=3.92, \mathrm{p}<0.01)$ (Table 1$)$.

\section{The difference in the plantar pressure and jerk valuesof the feet}

The difference in plantar pressure between the left foot and right foot in the $\mathrm{ADHD}$ group was greater than that observed in the control group $(\mathrm{z}=3.9, \mathrm{p}<0.01)$. The jerk score of the COP within the right foot in the $\mathrm{ADHD}$ group was higher than that observed in the control group $(\mathrm{z}=2.28, \mathrm{p}=0.02)$ (Table 2).

\section{Brain functional connectivity between the vermis of the cerebellum and other brain areas}

In the control group, the cerebellum was positively connected with the right parietal postcentral gyrus, right precuneus gyrus, right paracentral lobule, right cingulate gyrus, and left insular cortex $\left(\mathrm{p}_{\mathrm{FDR}<0.05}<0.005\right)$. In the $\mathrm{ADHD}$ group, the cere- bellum was positively connected with the right parahippocampal gyrus and the left temporal lobe $\left(\mathrm{p}_{\mathrm{FDR}<0.05}<0.004\right)$ (Figure 1, Table 3). There was no statistically significant correlation between K-ARS, jerk value, and brain activity.

A higher FC between the cerebellum and the right middle frontal gyrus (premotor cortex) and the medial frontal gyrus (cingulate gyrus) was observed in the control group as compared to the ADHD group $\left(\mathrm{p}_{\mathrm{FDR}<0.05}<0.04\right)$ (Figure 1, Table 3).

\section{Correlation between K-ARS score, plantar pressure, jerk value, and brain activity}

The K-ARS score was positively correlated with the difference in plantar pressure in all participants (ADHD and controls participants; $\mathrm{r}=0.55, \mathrm{p}<0.01$ ). However, the K-ARS score in the ADHD group showed no significant correlation with the difference in plantar pressure $(r=0.35, \mathrm{p}=0.23)$.

The difference in plantar pressure between the left and right foot in all participants (ADHD and control participants) was negatively correlated with the beta-value within the middle frontal gyrus (premotor cortex; $r=-0.50, p=0.01$ ). In the ADHD group, the difference in plantar pressure between the left and right feet was also negatively correlated with the beta-value within the middle frontal gyrus (premotor cortex; $r=-0.57$,

Table 1. Demographic characteristics

\begin{tabular}{lccc}
\hline & ADHD group & Control group & Statistics \\
\hline Age & $10.7 \pm 1.4$ & $10.4 \pm 1.2$ & $\mathrm{z}=0.48, \mathrm{p}=0.63$ \\
Sex (male/female) & $10 / 3$ & $9 / 4$ & $\chi^{2}=0.2, \mathrm{p}=0.68$ \\
Years of education & $4.5 \pm 1.2$ & $4.3 \pm 1.0$ & $\mathrm{z}=0.48, \mathrm{p}=0.63$ \\
IQ & $96.0 \pm 9.7$ & $96.2 \pm 8.3$ & $\mathrm{z}=0.39, \mathrm{p}=0.70$ \\
Height $(\mathrm{cm})$ & $145.3 \pm 13.4$ & $147.2 \pm 7.2$ & $\mathrm{z}=0.31, \mathrm{p}=0.76$ \\
Weight $(\mathrm{kg})$ & $46.1 \pm 14.2$ & $45.1 \pm 11.1$ & $\mathrm{z}=0.10, \mathrm{p}=0.63$ \\
K-ARS & $20.4 \pm 11.9$ & $5.0 \pm 3.6$ & $\mathrm{Z}=3.39, \mathrm{p}<0.01^{*}$ \\
Inatt/Hyper/Combined & $4 / 6 / 3$ & - & - \\
CDI & $9.5 \pm 6.0$ & $7.1 \pm 6.1$ & $\mathrm{z}=0.87, \mathrm{p}=0.38$ \\
\hline
\end{tabular}

*statistically significant. IQ: intelligence quotient, K-ARS: Korean attention-deficit/hyperactivity disorder (ADHD) scale, Inatt: predominantly inattentive presentation, Hyper: predominantly hyperactive/impulsive presentation, Combined: combined presentation, CDI: children's depressive inventory

Table 2. Differencesin plantar pressure and jerk value of the feet

\begin{tabular}{lccc}
\hline & ADHD & Control & Statistics \\
\hline Plantar pressure & & & $\mathrm{z}=2.24, \mathrm{p}=0.03^{*}$ \\
$\quad$ Left foot & $48.3 \pm 1.4$ & $49.2 \pm 1.1$ & $\mathrm{z}=2.24, \mathrm{p}=0.03^{*}$ \\
$\quad$ Right foot & $51.7 \pm 1.4$ & $50.8 \pm 1.1$ & $\mathrm{z}=3.92, \mathrm{p}<0.01^{*}$ \\
$\quad$ Difference & $4.0 \pm 1.8$ & $2.1 \pm 1.6$ & $\mathrm{z}=1.18, \mathrm{p}=0.23$ \\
Jerk value of COP & & & $\mathrm{z}=2.28, \mathrm{p}=0.02^{*}$ \\
$\quad$ Left foot & $1.5 \pm 0.4$ & $1.3 \pm 0.2$ & $1.2 \pm 0.2$ \\
Right foot & $1.5 \pm 0.3$ & & \\
\hline
\end{tabular}

*statistically significant. ADHD: attention-deficit/hyperactivity disorder, COP: center of pressure 
Table 3. Brain functional connectivity between the vermis of thecerebellum and other brain areas

\begin{tabular}{|c|c|c|c|c|c|}
\hline \multicolumn{3}{|c|}{ Talairach code } & \multirow{2}{*}{ CLs } & \multirow{2}{*}{$\mathrm{p}_{\mathrm{FDR}<0.05}$} & \multirow{2}{*}{ Regions } \\
\hline $\mathrm{x}$ & $\mathrm{y}$ & $\mathrm{Z}$ & & & \\
\hline \multicolumn{6}{|c|}{ Control children } \\
\hline 49 & -11 & 17 & 128 & 0.005 & 1. Right Parietal Postcentral Gyrus, BA 43 \\
\hline 5 & -18 & -21 & 132 & 0.005 & 2. Right Brainstem, Pons \\
\hline 2 & -74 & 39 & 127 & 0.005 & 3. Right Parietal Precuneus, BA7 \\
\hline 4 & -21 & 43 & 121 & 0.005 & 4. Right Frontal, Paracentral Lobule, BA 31 \\
\hline 1 & 24 & 33 & 120 & 0.005 & 5. Right Cingulate Gyrus, BA 32 \\
\hline-43 & -34 & 22 & 129 & 0.005 & 6. Left Insula, BA 13 \\
\hline \multicolumn{6}{|c|}{ ADHD children } \\
\hline 2 & -60 & -17 & 117 & 0.004 & 7. Right Cerebellum, Posterior Lobe \\
\hline-39 & -7 & -15 & 108 & 0.004 & 8. Left Temporal Lobe, BA 20 \\
\hline 34 & -7 & -13 & 125 & 0.004 & 9. Right, Parahippocampal Gyrus, Amygdala \\
\hline \multicolumn{6}{|c|}{ Control children $>$ ADHD children } \\
\hline 29 & 21 & 42 & 42 & 0.04 & 10. Right Middle Frontal Gyrus, BA 8 \\
\hline 2 & 47 & 11 & 41 & 0.04 & 11. Right Medial Frontal Gyrus, BA 10 \\
\hline
\end{tabular}

CLs: clusters, BA: Broadmann area, ADHD: attention-deficit/hyperactivity disorder

$\mathrm{p}=0.04$ ). However, the control group did not show a significant correlation between the difference in plantar pressure and the beta-value within the middle frontal gyrus (premotor cortex; $\mathrm{r}=0.22, \mathrm{p}=0.48$ ).

\section{DISCUSSION}

The current study indicated that children with ADHD exhibit disturbance of balance as assessed by plantar pressure. In addition, disturbance of balance in children with ADHD was associated with decreased brain connectivity within the premotor cortex. To the best of our knowledge, this is the first study on ADHD to show a correlation between balance as assessed by plantar pressure and activity within the brain regions controlling balance.

\section{The difference in plantar pressure and jerk value of the feet}

Our study suggests that children with ADHD exhibit disturbance of balance as assessed by plantar pressure. In addition, the severity of ADHD symptoms was correlated with body imbalance. The disturbance of balance control in children with ADHD has been reported in previous studies. ${ }^{22}$ Buderath et al. ${ }^{23}$ reported that children with ADHD showed difficulties in a backward walking test and a paced stepping test. However, Schlee et al. ${ }^{6}$ reported that the balance and postural capacity (plantar foot sensitivity and static balance) of children with ADHD were not different from those of controls. The differing results between our study and Schlee's study may be due to the effect of medication. Thirteen of the 21 children with ADHD in Schlee's study were on medication at the time of assessment, while the children with ADHD in the current study were drug-naïve. Methylphenidate is thought to affect postural control in children with ADHD. ${ }^{24}$ Taken together, we cautiously suggest that when compared to controls, children with ADHD have balance disturbance as assessed by plantar pressure.

\section{Brain functional connectivity between the cerebellum and other brain areas}

In the current study, the control children showed FC from the cerebellum to the parietal, cingulate, and insular cortices, while children with ADHD showed FC from the cerebellum to just the temporal area. The parieto-temporal junction and the parieto-insular cortex have been thought to be associated with static and dynamic balance. ${ }^{25,26}$ In particular, postural stabilization has been reported to be associated with activation within the parietal lobe, anterior cingulate cortex, and cerebellum. ${ }^{27}$

When directly comparing brain connectivity from the cerebellum to other brain areas, children with ADHD showed decreased connectivity from the cerebellum to the middle frontal gyrus (premotor cortex) and medial frontal gyrus (anterior cingulate) as compared to the control children. The cerebellum is already known to play a crucial role in movement control, balance, and locomotion. ${ }^{28}$ Karim et al. ${ }^{29}$ reported that the middle frontal gyri and anterior cingulate were associated with anterior-to-posterior movement in the COP in healthy adults. More interestingly, the degree of balance disturbances in the current study was associated with decreased brain activity within the premotor cortex inchildren with ADHD. Taken to- 
gether, we suggest that decreased brain connectivity from the cerebellum to the middle frontal and anterior cingulate gyri disturbs balance and posture in children with $\mathrm{ADHD}$.

\section{Limitations}

There were several limitations to the current research. First, the small number of participants means that we cannot generalize the results. Second, only the balance in static and simple tasks (e.g., walking forward) was assessed in the current research. Additional studies with a large cohort of participants and complex movements are needed.

\section{Conclusion}

Children with ADHD exhibit disturbance of balance as assessed by plantar pressure. Decreased brain connectivity from the cerebellum to the middle frontal and medial frontal gyri in children with ADHD was associated with disturbances of posture and balance.

\section{Acknowledgments}

This study was supported by a grant from the Korean Health Technology R\&D Project, Ministry of Health \& Welfare, Republic of Korea (A120013) and a grant from the Korea Creative Content Agency (R2014040055) in the collection, analysis and interpretation of data and in the writing of the manuscript.

\section{REFERENCES}

1. Ghanizadeh A. Predictors of postural stability in children with ADHD. J Atten Disord 2011;15:604-610.

2. Kolb B, Gibb R. Brain plasticity and behaviour in the developing brain. J Can Acad Child Adolesc Psychiatry 2011;20:265-276.

3. Biederman J, Faraone SV. Attention-deficit hyperactivity disorder. Lancet 2005;366:237-248.

4. Tervo RC, Azuma S, Fogas B, Fiechtner H. Children with ADHD and motor dysfunction compared with children with ADHD only. Dev Med Child Neurol 2002;44:383-390.

5. Konicarova J, Bob P, Raboch J. Balance deficits and ADHD symptoms in medication-naive school-aged boys. Neuropsychiatr Dis Treat 2014; 10:85-88.

6. Schlee G, Neubert T, Worenz A, Milani TL. Children with ADHD show no deficits in plantar foot sensitivity and static balance compared to healthy controls. Res Dev Disabil 2012;33:1957-1963.

7. Rubia K, Overmeyer S, Taylor E, Brammer M, Williams SC, Simmons A, et al. Hypofrontality in attention deficit hyperactivity disorder during higher-order motor control: a study with functional MRI. Am J Psychiatry 1999;156:891-896.

8. O'Halloran CJ, Kinsella GJ, Storey E. The cerebellum and neuropsychological functioning: a critical review. J Clin Exp Neuropsychol 2012;34:35-56.

9. Rubia K, Overmeyer S, Taylor E, Brammer M, Williams SC, Simmons A, et al. Hypofrontality in attention deficit hyperactivity disorder during higher-order motor control: a study with functional MRI. Am J
Psychiatry 1999;156:891-896.

10. Zang YF, Jin Z, Weng XC, Zhang L, Zeng YW, Yang L, et al. Functional MRI in attention-deficit hyperactivity disorder: evidence for hypofrontality. Brain Dev 2005;27:544-550.

11. Seidman LJ, Valera EM, Makris N. Structural brain imaging of attention-deficit/hyperactivity disorder. Biol Psychiatry 2005;57:1263-1272.

12. Timmann D, Diener HC. Coordination and Ataxia. Orlando: Anders; 2003.

13. Mackey DC, Robinovitch SN. Postural steadiness during quiet stance does not associate with ability to recover balance in older women. Clin Biomech (Bristol, Avon) 2005;20:776-783.

14. Blaszczyk JW, Bacik B, Juras G. Clinical assessment of postural stability. J Mech Med Biol 2003;3:135-144.

15. Rosenbaum D, Becker H. Plantar pressure distribution measurements. Technical background and clinical applications. Foot Ankle Surg 1997; 3:1-14.

16. Cousins SD, Morrison SC, Drechsler WI. The reliability of plantar pressure assessment during barefoot level walking in children aged 7-11 years. J Foot Ankle Res 2012;5:8.

17. Kim YS, Cheon KA, Kim BN, Chang SA, Yoo HJ, Kim JW, et al. The reliability and validity of Kiddie-Schedule for Affective Disorders and Schizophrenia-Present and Lifetime Version-Korean version (K-SADSPL-K). Yonsei Med J 2004;45:81-89.

18. Hass CJ, Waddell DE, Wolf SL, Juncos JL, Gregor RJ. Gait initiation in older adults with postural instability. Clin Biomech (Bristol, Avon) 2008; 23:743-753.

19. Bryant A, Singer K, Tinley P. Comparison of the reliability of plantar pressure measurements using the two-step and midgait methods of data collection. Foot Ankle Int 1999;20:646-650.

20. Talairach J, Tournoux P. Co-Planar Stereotactic Atlas of the Human Brain. New York: Thieme Medical Publishers, Inc,; 1988.

21. Biswal BB, Van Kylen J, Hyde JS. Simultaneous assessment of flow and BOLD signals in resting-state functional connectivity maps. NMR Biomed 1997;10:165-170.

22. Shum SB, Pang MY. Children with attention deficit hyperactivity disorder have impaired balance function: involvement of somatosensory, visual, and vestibular systems. J Pediatr 2009;155:245-249.

23. Buderath P, Gartner K, Frings M, Christiansen H, Schoch B, Konczak $\mathrm{J}$, et al. Postural and gait performance in children with attention deficit/hyperactivity disorder. Gait Posture 2009;29:249-254.

24. Bucci MP, Seassau M, Larger S, Bui-Quoc E, Gerard CL. Effect of visual attention on postural control in children with attention-deficit/hyperactivity disorder. Res Dev Disabil 2014;35:1292-1300.

25. Perennou DA, Leblond C, Amblard B, Micallef JP, Rouget E, Pelissier J. The polymodal sensory cortex is crucial for controlling lateral postural stability: evidence from stroke patients. Brain Res Bull 2000;53:359-365.

26. Ustinova KI, Chernikova LA, Ioffe ME, Sliva SS. Impairment of learning the voluntary control of posture in patients with cortical lesions of different locations: the cortical mechanisms of pose regulation. Neurosci Behav Physiol 2001;31:259-267.

27. Slobounov $\mathrm{S}$, Wu T, Hallett M. Neural basis subserving the detection of postural instability: an fMRI study. Motor Control 2006;10:69-89.

28. Morton SM, Bastian AJ. Mechanisms of cerebellar gait ataxia. Cerebellum 2007;6:79-86.

29. Karim HT, Sparto PJ, Aizenstein HJ, Furman JM, Huppert TJ, Erickson KI, et al. Functional MR imaging of a simulated balance task. Brain Res 2014;1555:20-27. 